\title{
OPTICAL OBSERVATIONS OF \\ IONIZED GAS IN EXTERNAL GALAXIES
}

\section{G. MONNET}

\author{
Observatoire de Marseille, Marseille, France
}

\begin{abstract}
This paper reviews recent optical results on the large scale distribution of ionized gas in spiral galaxies, including our own. There is a diffuse, inhomogeneous emission in the arm region in spirals, including our Galaxy, and in gas-rich galaxies a fainter diffuse emission between the arms.
\end{abstract}

\section{Ionized Gas in External Galaxies}

Surveys of the ionized gas in external galaxies show three major distributions on a large scale:

(i) The classical $\mathrm{H}$ II regions, mainly located in the spiral arms, as is well known. The emission measures (computed throughout this paper from the $\mathrm{H} \alpha$ line intensity for a temperature of $7500 \mathrm{~K}$ ) are typically a few times $10^{3} \mathrm{~cm}^{-6} \mathrm{pc}$.

(ii) A diffuse arm emission which connects the $\mathrm{H}$ II regions. It can be seen easily on plates taken in $\mathrm{H} \alpha$ light through an interference filter for a number of gas-rich galaxies, i.e., of $S_{\mathrm{c}}$ to $S_{\mathrm{d}}$ Hubble types, for instance in M33, M101, M83, ... (Monnet, 1971). On the nearest $S_{\text {cd }}$ galaxy, M33, this emission exhibits a clear filamentary structure (Figure 1), which a scale of $\sim 100 \mathrm{pc}$.

The same phenomenon - with an emission measure of $\sim 100 \mathrm{~cm}^{-6} \mathrm{pc}$ - has been found in the nearest $S_{\mathrm{b}}$ galaxy, M31, by Deharveng and Pellet (1970) (Figure 2).

The intensity ratios between $\mathrm{H} \alpha 6563 \AA$ and [N II] $6584 \AA$ have been studied in M33 by Benvenuti et al. (1973) and by Comte (1974) (in preparation) with similar conclusions. Results by Comte are shown in Figure 3; the $\mathrm{H} \alpha /[\mathrm{N} \mathrm{II}]$ ratio is fairly normal in the diffuse arm emission, $\sim 5$. I have made a rough study of the $\mathrm{H} \alpha /[\mathrm{N} \mathrm{II}]$ ratio in M31 from a comparison of the $\mathrm{H} \alpha$ plate shown in Figure 2 and a [N II] plate taken in the same field. The ratios appear also quite normal in the diffuse arm emission. These results indicate that the ionization is probably normal, similar to that of the more condensed $\mathrm{H}$ II regions.

(iii) A diffuse emission in the disk, between the arms. The first detection of a disk emission was made by Mayall and Aller (1939) in M33, in the [O II] $3727 \AA$ doublet, and the first two dimensional study by Carranza et al. (1968) likewise in M33, but in the $\mathrm{H} \alpha$ and $[\mathrm{NII}] 6584 \AA$ lines. So far disk emissions have been detected - with emission measures around $30 \mathrm{~cm}^{-6} \mathrm{pc}$ - only in the central part ( 2 to $3 \mathrm{kpc}$ diameter) of $S_{\mathrm{c}}$ to $S_{\mathrm{d}}$ type galaxies, marginally detected in M51 by Tully (1974) and not at all in the $S_{\mathrm{b}}$ spiral M31. In M33, the $\mathrm{H} \alpha$ to [N II] ratio is abnormal, $\sim 1.5$, which can be explained in terms of ionization by late blue stars - either normal B stars or the dwarf blue stars hypothetized by Hills (1972) - or with more exotic ionization sources, as hard UV, soft X-rays or MeV protons. For a clearcut answer one needs a sufficient number of line intensity ratios, as the theoretical values in each case have been com- 


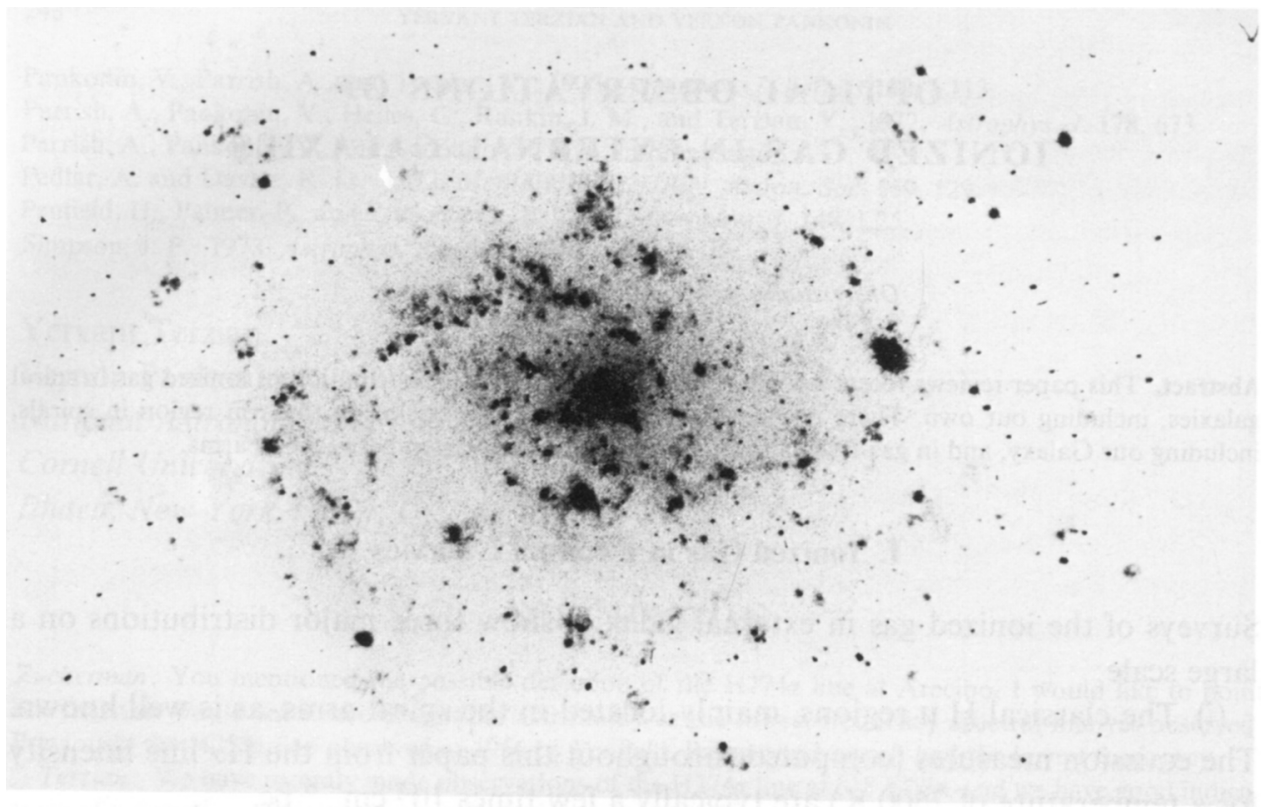

Fig. 1. H $\alpha$ filter photograph of M33 over a $40^{\prime}$ field made with the $193 \mathrm{~cm}$ telescope at Haute Provence Observatory. North is at right. Note the diffuse filamentary emission in the arms. The disk emission is too weak to be seen on a photograph and can be only detected with spectrographs.

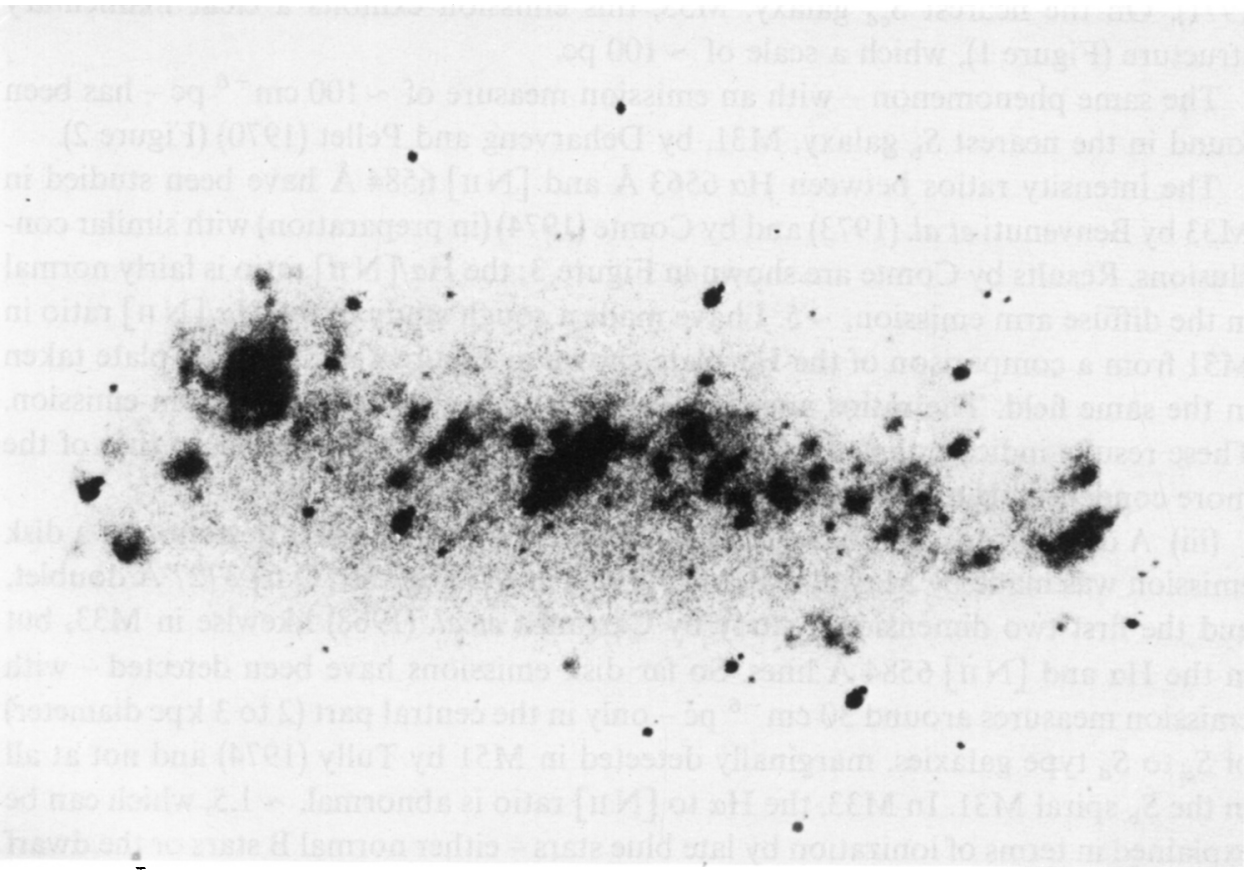

Fig. 2. $\mathrm{H} \alpha$ filter photograph of $\mathrm{M} 31$ showing a $15^{\prime}$ length of the $\mathrm{N}_{4}$ Spiral arm (Baade and Arp's notation). Note the diffuse filamentary emission in the arm between the classical $\mathrm{H}$ il regions. The $\mathrm{H}$ II regions are much fainter than in Sivan's atlas of our Galaxy made with a roughly similar scale in parsecs, showing that our Galaxy is more likely a $S_{\mathrm{bc}}-\mathrm{S}_{\mathrm{c}}$ than a $S_{\mathrm{b}}$. 


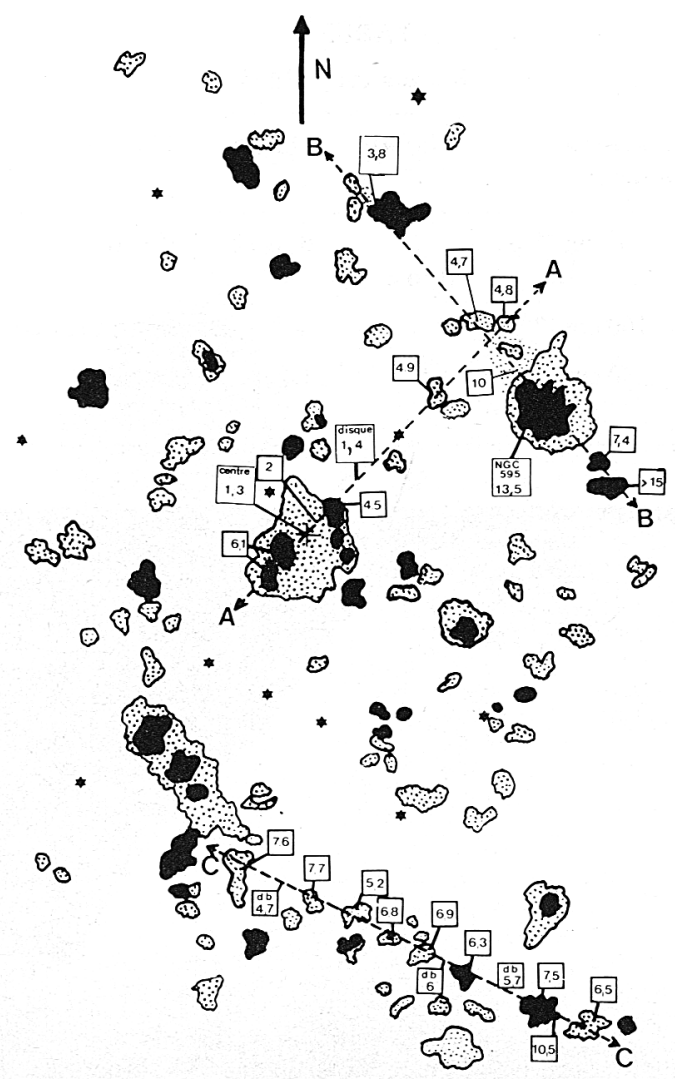

Fig. 3. Map of the $\mathrm{H} \alpha /[\mathrm{N}$ II $]$ ratios on a $15^{\prime}$ field centered on M33, obtained by Comte with a Cassegrain spectrograph attached to the $193-\mathrm{cm}$ telescope at Haute Provence Observatory.

Spectra are labeled $A, B$ and $C$. Note the low values 1.4 in the disk emission (disk) and in the very central part of the galaxy (Spectrum A). Note the high values 4.7, 5.7, and 6 on the diffuse arm emission (db) on Spectrum C.

puted for the most common lines by Bergeron and Souffrin (1971). Benvenuti et al. (1973) have discovered a faint [S II] emission in the disk of M33, with an abnormal $\mathrm{H} \alpha /[\mathrm{S} \mathrm{II}]$ ratio $\sim 1.5$ (instead of 10 for classical $\mathrm{H}$ II regions). A similar result has been obtained by Comte (1974) (in preparation), who has also observed $\mathrm{H} \beta$ and an exceedingly faint [O III] $5007 \AA$ emission, but with a probability of positive detection of only $80 \%$. These data are summarized in Table I. The faintness of the [O III] line definitely excludes ionization by hard UV or soft X-rays and leaves moderately blue stars or $\mathrm{MeV}$ protons as the only candidates.

\section{Ionized Gas in Our Galaxy}

Judging from the large scale distribution in external galaxies, one can expect in our Galaxy - whose Hubble type is most probably in the $S_{\mathrm{bc}}, S_{\mathrm{c}}$ range - a diffuse arm 
TABLE I

Ionized gas in M33

\begin{tabular}{|c|c|c|c|c|c|}
\hline & $\begin{array}{l}\text { Emission } \\
\text { measure }\end{array}$ & $\mathrm{H} \alpha / \mathrm{N}_{\text {II }}$ & $\mathrm{H} \alpha / \mathrm{S}_{\text {II }}$ & $\mathrm{H} \beta / \mathrm{O}$ III & Ionization \\
\hline Classical H II regions & $>10^{3} \mathrm{~cm}^{-6} \mathrm{pc}$ & 4 to 8 & 8 to 12 & 0.3 to 1 & O stars \\
\hline Diffuse arm emission & $100 \mathrm{~cm}^{-6} \mathrm{pc}$ & & $>5$ & not measured & $\begin{array}{l}\text { probably } \\
\text { O stars }\end{array}$ \\
\hline Disk emission & $30 \mathrm{~cm}^{-6} \mathrm{pc}$ & 1.5 & 1.5 & $\leqslant 4$ & $\begin{array}{l}\text { B stars or } \\
\text { MeV protons }\end{array}$ \\
\hline
\end{tabular}

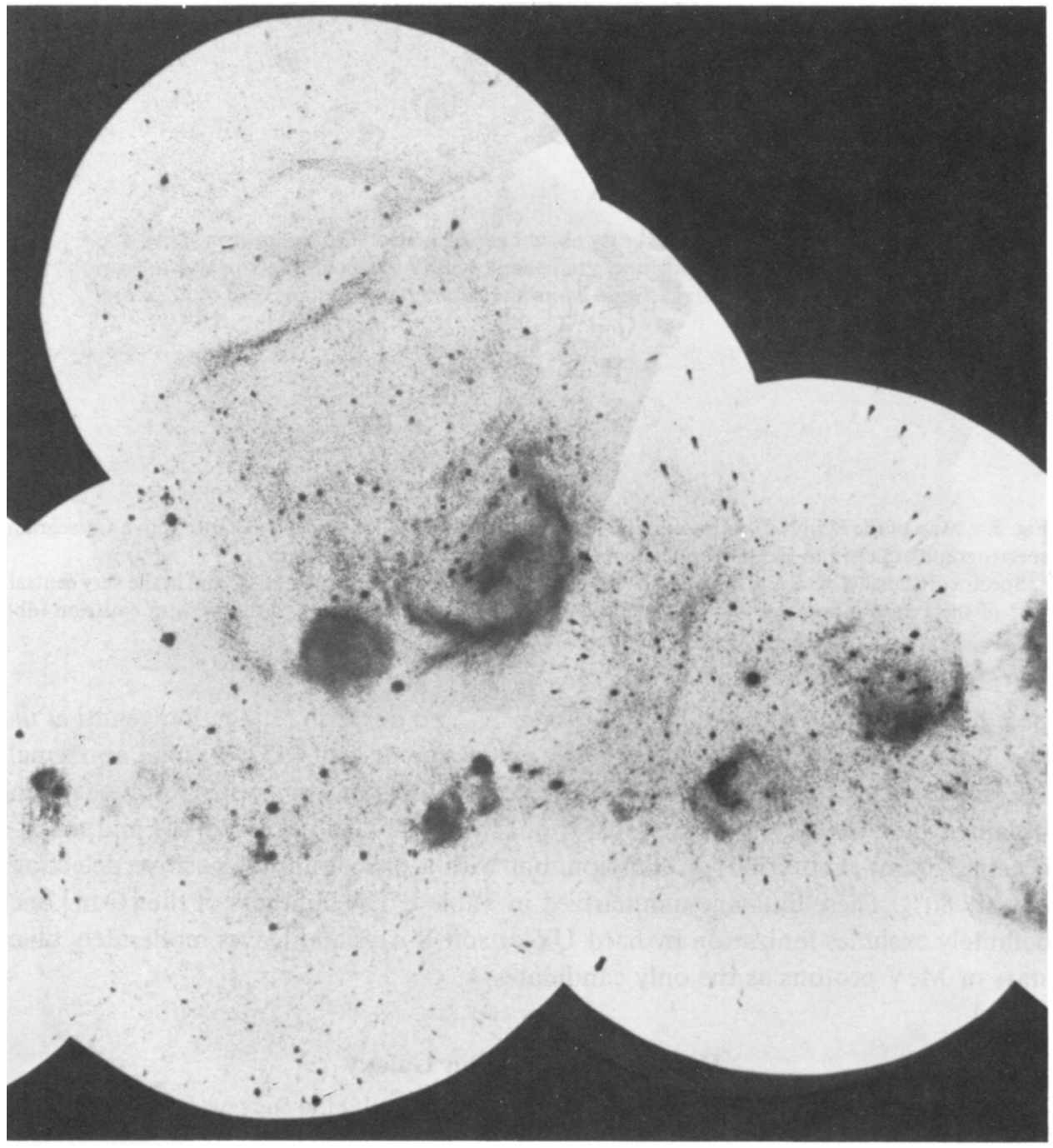

Fig. 4. H $\alpha$ photograph over an $85^{\circ}$ field in the Orion region. One sees filaments over a $60^{\circ}$ field. The strongest filament at top has been discovered by Meaburn (1965) and interpreted by him as an optical counterpart of the Cetus arc. The two large nebulae at the center of the plate are $\lambda$ Orionis and the Orion loop. 


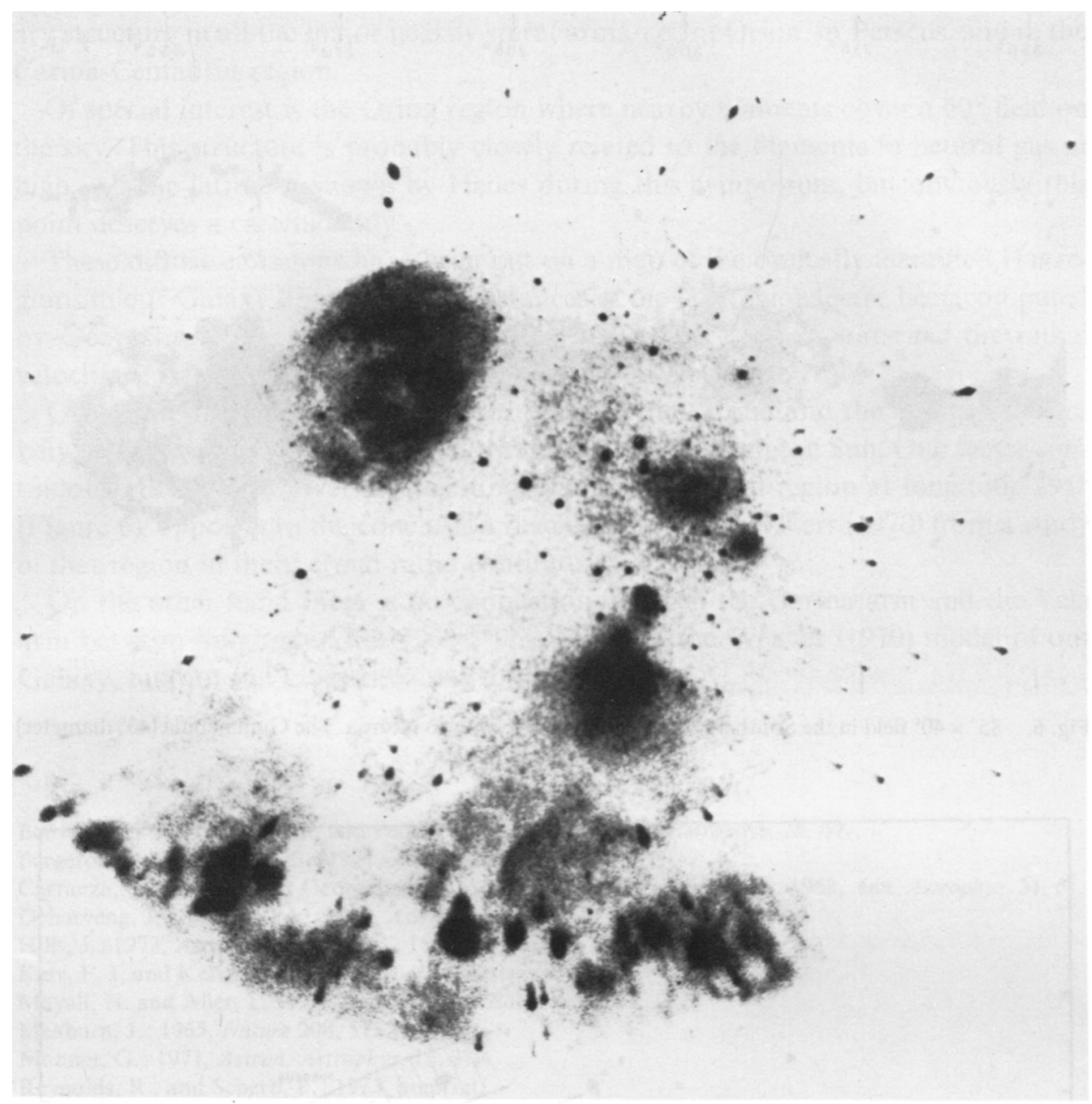

Fig. 5. $\mathrm{H} \alpha$ photograph over a $40^{\circ}$ field in Sagittarius. At bottom a portion of the Sagittarius arm. Top left, the $\zeta$ Ophiuchi nebula. Top right a new shell nebula in Scorpius. The position and the shape of this nebula are strikingly similar to a neutral hydrogen shell found by Sancisi and presented during this symposium.

emission $\sim 100 \mathrm{~cm}^{-6} \mathrm{pc}$ (perpendicular to the plane). This emission has been observed by various authors in radio recombination lines (see Guelin, 1974) and quite recently in optical lines by Reynolds and Scherb (1973).

On the other hand, if there is any disk emission it is probably very faint (a few $\mathrm{cm}^{-6} \mathrm{pc}$ ), quite close to the center, and of course severely blended with the stronger emission in the arm.

A near whole-sky survey in $\mathrm{H} \alpha$ has been made by Sivan with a small diameter $(1 \mathrm{~cm})$, large field $\left(55^{\circ}\right)$ and high aperture ratio $(F / 1)$ telescope. Part of this survey is shown in Figures 4, 5 and 6. There is a clear diffuse arm emission with some filament- 


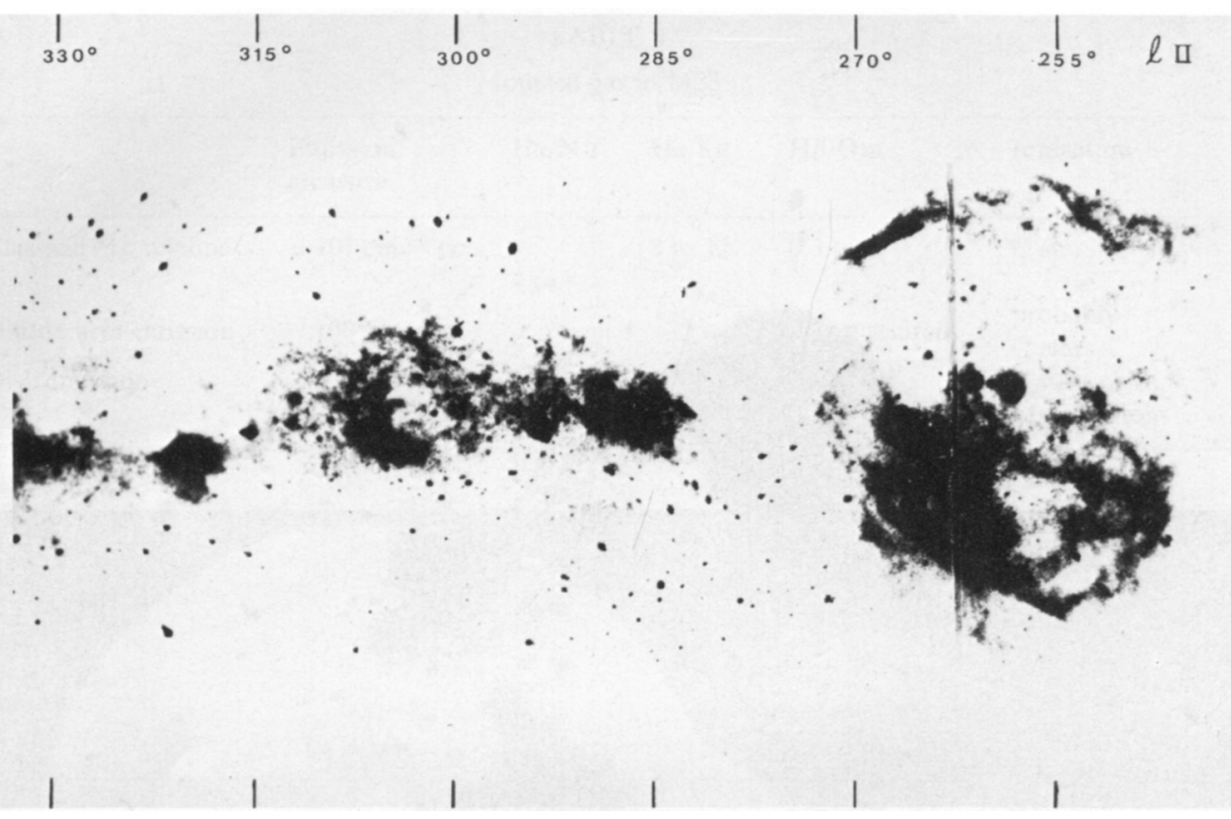

Fig. 6. $85^{\circ} \times 40^{\circ}$ field in the Southern Milky Way from Puppis to Norma. The Gum nebula ( $40^{\circ}$ diameter) is at right. North is at top.

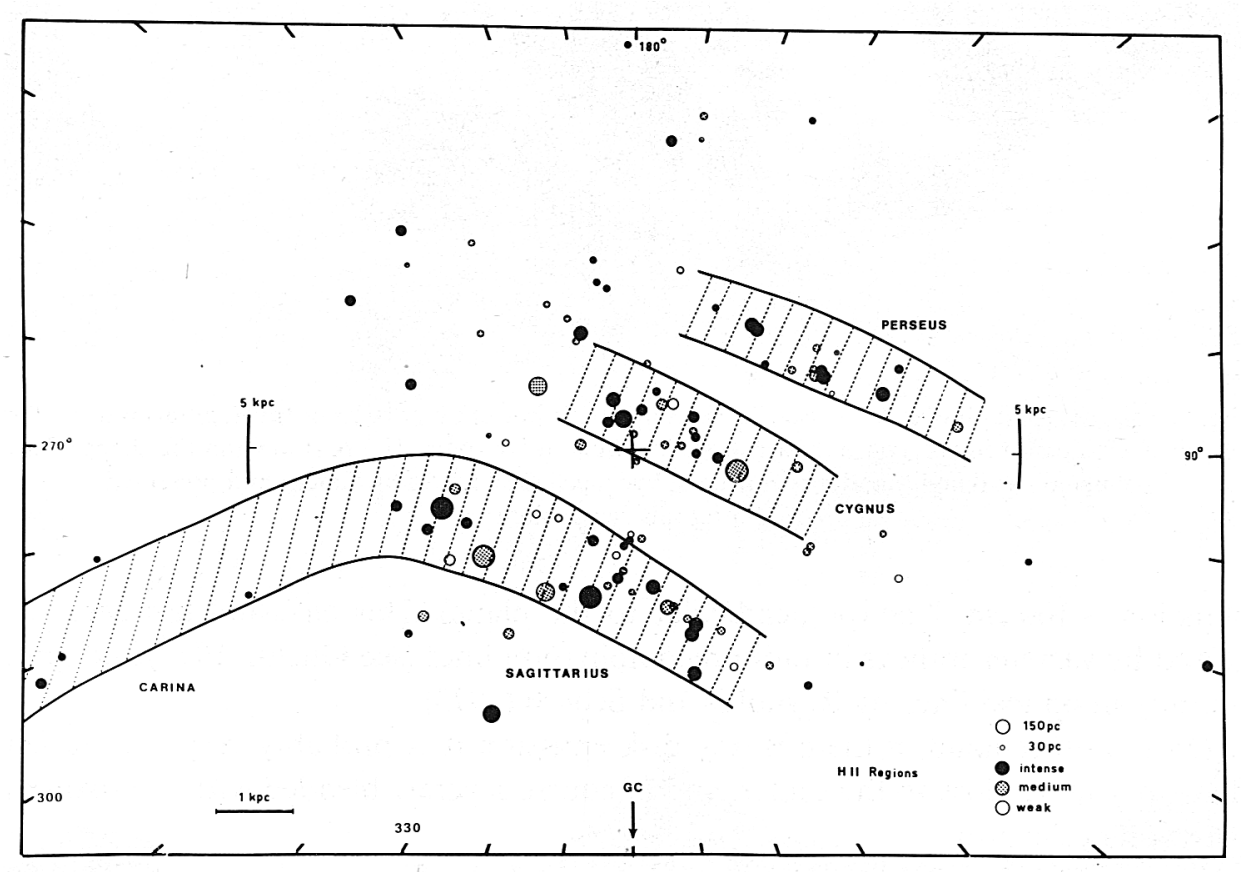

Fig. 7. Map of the optically identified $\mathrm{H}$ II regions. Strong to weak $\mathrm{H}$ II regions are indicated by circles with three different intensities and with their real diameter. The regions of continuous arm emission are shaded. 
ary structure in all the major nearby spiral arms, i.e., in Orion, in Perseus, and in the Carina-Centaurus region.

Of special interest is the Orion region where nearby filaments cover a $60^{\circ}$ field on the sky. This structure is probably closely related to the filaments in neutral gas at high galactic latitudes shown by Heiles during this symposium, but obviously this point deserves a careful study.

These diffuse emissions have been put on a map of the optically identified $\mathrm{H}$ II regions in our Galaxy (Figure 7). The distances of the $\mathrm{H}$ II regions have been computed by Georgelin, using the photometric distance of the exciting stars and the radial velocities measured with a Fabry-Pérot interferometer.

Of special interest is the connection between the Carina and the Sagittarius arm between longitudes $292^{\circ}-304^{\circ}$ at a distance $\sim 1.5 \mathrm{kpc}$ from the Sun. One sees a continuous $\mathrm{H} \alpha$ emission over all this range and a strong $\mathrm{H}$ II region at longitude $295^{\circ}$ (Figure 6), opposite to the conclusion reached by Kerr and Kerr (1970) from a study of that region in the thermal radio continuum.

On the other hand there is no connection between the Carina arm and the Vela arm between longitudes $282^{\circ}-268^{\circ}$. This supports the Weaver (1970) model of our Galaxy, but not the Leiden-Sydney model.

\section{References}

Benvenuti, P., D'Odorico, S., and Peimbert, M.: 1973, Astron. Astrophys. 28, 447.

Bergeron, J. and Souffrin, S.: 1971, Astron. Astrophys. 14, 167.

Carranza, G., Courtès, G., Georgelin, Y., Monnet, G., and Pourcelot, A.: 1968, Ann. Astrophys. 31, 61.

Deharveng, J. and Pellet, A.: 1970, Astron. Astrophys. 9, 181.

Hills, J.: 1972, Astron. Astrophys. 17, 155.

Kerr, F. J. and Kerr, M.: 1970, Astrophys. Letters 6, 175.

Mayall, N. and Aller, L.: 1939, Publ. Astron. Soc. Pacific 51, 112.

Meaburn, J. : 1965, Nature 208, 575.

Monnet, G.: 1971, Astron. Astrophys. 12, 379.

Reynolds, R., and Scherb, F.: 1973, preprint.

Tully, B.: 1974, Astrophys. J. Suppl. $27,415$.

Weaver, H.: 1970, in W. Becker and G. Contopoulos (eds.), 'The Spiral Structure of Our Galaxy', IAU Symp. 38, 126.

\section{G. Monnet}

Observatoire de Marseille,

2, Place Le Verrier,

F-13004 Marseille, France

\section{DISCUSSION}

Baldwin: What is the value of $\left\langle n_{\mathrm{e}}^{2}\right\rangle$ which you deduce from your observations of the distributed $\mathrm{H} \alpha$ emission in the arms of our Galaxy? And is this value consistent with the values of $\left\langle n_{\mathrm{e}}\right\rangle$ obtained from pulsar dispersion measures?

Monnet: With an emission measure of about $100 \mathrm{~cm}^{-6} \mathrm{pc}$ and a typical size of about $100 \mathrm{pc}$, the value of $\left\langle n_{\mathrm{e}}^{2}\right\rangle^{1 / 2}$ is of the order of $1 \mathrm{~cm}^{-3}$. If it was homogeneously distributed $\left\langle n_{\mathrm{e}}\right\rangle$ would be equal to 
1 , too much to fit with the values given by the dispersion measures on pulsars. But it is clear that the ionized gas distribution is highly nonhomogeneous and there is probably no large conflict.

Burton: What is the scale height of the distributed emission in our Galaxy and in M33?

Monnet: In our Galaxy, the continuous arm emission height can be measured directly from the plates. It gives about $200 \mathrm{pc}$, similar to that of the classical Hil regions. In M33 both continuous arm emission and continuous disk emission heights can only be inferred from the rms radial velocity dispersion, which is about $6 \mathrm{~km} \mathrm{~s}^{-1}$ - even smaller than that of the classical $\mathrm{HII}$ regions. The scale height must be thus similar, $\sim 200 \mathrm{pc}$.

Mezger: Could you define what you mean by "low excitation ionized gas in the disk?"

Monnet: We see only line emission from ions whose ionization potentials are very similar to hydrogen, i.e., $\mathrm{N}^{+}, \mathrm{S}^{+}$and probably $\mathrm{O}^{+}$but practically no $\mathrm{O}^{++}$.

Terzian: How do you arrive at the lower limit of the electron density from your emission measure values? Do you assume a filamentary structure for the diffuse interstellar medium?

Monnet: If there was no filling factor $n_{\mathrm{e}}$ would be $\sim 1 \mathrm{~cm}^{-3}$ just by dividing the emission measure by the scale height. This, of course, is only a lower limit, especially because we know that there is filamentary structure. 